\title{
Brucellosis Reemergence after a Decade of Quiescence in Palestine, 2015-2017: a Seroprevalence and Molecular Characterization Study
}

\author{
Bessan Aljanazreh ${ }^{1}$, Khaled Alzatari ${ }^{1}$, Asmaa Tamimi ${ }^{1}$, Mohammad Alsaafeen ${ }^{2}$, Waheed \\ Hassouneh $^{3}$, and Yaqoub Ashhab ${ }^{1}$ \\ ${ }^{1}$ Palestine Polytechnic University \\ ${ }^{2}$ Palestinian Ministry of Health \\ ${ }^{3}$ Palestine Red Crescent Specialized Hospital
}

March 16, 2021

\begin{abstract}
Brucellosis is an endemic disease in many developing countries and ranked by the World Health Organization among the top seven "neglected zoonoses". Although a Palestinian brucellosis control program was launched in 1998, the disease reemerged after 2012. Interestingly, a similar reemerging pattern was reported in the neighboring Israeli regions. The aim of this work was to characterize the reemerging strains and delineate their genetic relatedness. During 2015-2017, blood samples from 1324 suspected patients were analyzed using two serological tests. Seropositive samples were cultured, and their DNAs were analyzed by different genetic markers to determine the involved Brucella species and rule out any possible involvement of the Rev.1 vaccine strain. The rpoB gene was sequenced from 9 isolates to screen for rifampicin-resistance mutations. Multi Locus VNTR Analysis (MLVA-16) was used for genotyping the isolates. The molecular analysis showed that all isolates were B. melitensis strains unrelated to the Rev.1 vaccine. The rpoB gene sequences showed four single nucleotide variations (SNVs) not associated with rifampicin resistance. MLVA-16 analysis clustered the isolates into 22 unique genotypes that belong to the East Mediterranean lineage. Altogether, our findings show that the reemergence of brucellosis was due to B. melitensis strains of local origin, the Palestinian and Israeli control programs' weaknesses could be a major factor behind the reemergence of the disease. However, other socioeconomic and environmental factors must be investigated. Moreover, strengthening brucellosis control programs and enhancing cooperation between all stakeholders is essential to ensure long-term program outcomes to fight brucellosis.
\end{abstract}

\section{Hosted file}

Brucellosis Reemergence after a Decade of Quiescence in Palestine, 2015-2017 a Seroprevalence and Molec available at https://authorea.com/users/401934/articles/513878-brucellosis-reemergenceafter-a-decade-of-quiescence-in-palestine-2015-2017-a-seroprevalence-and-molecularcharacterization-study 

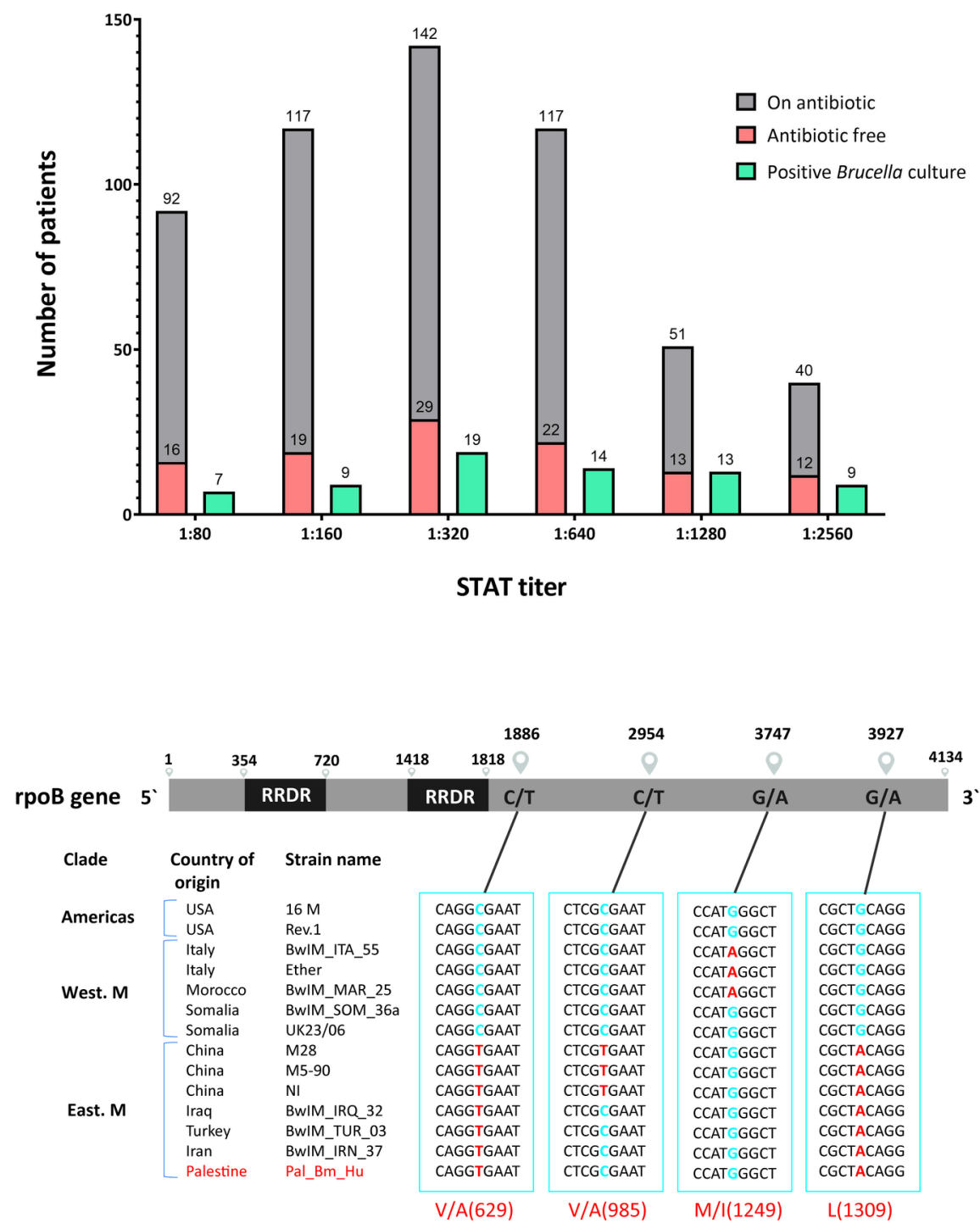


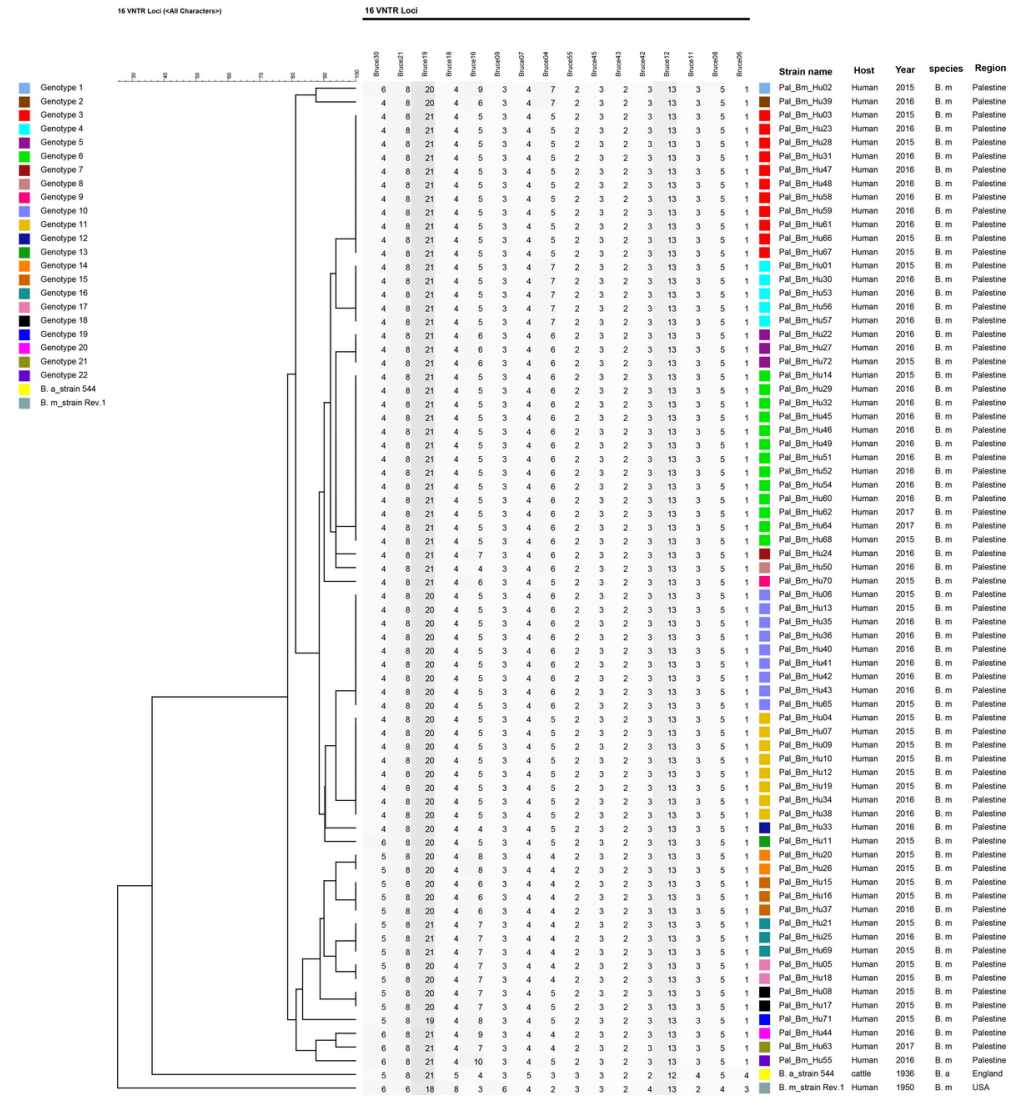




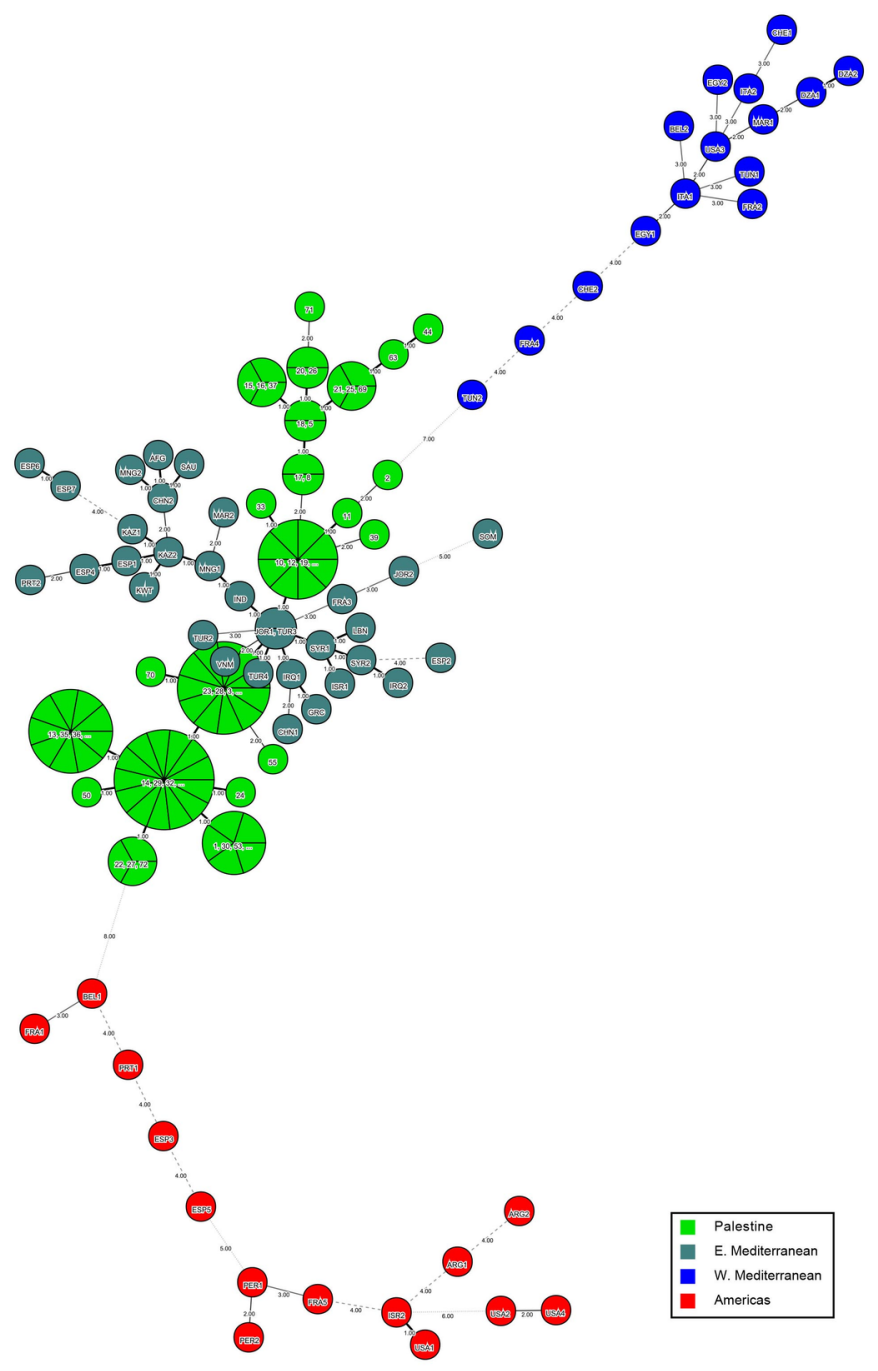




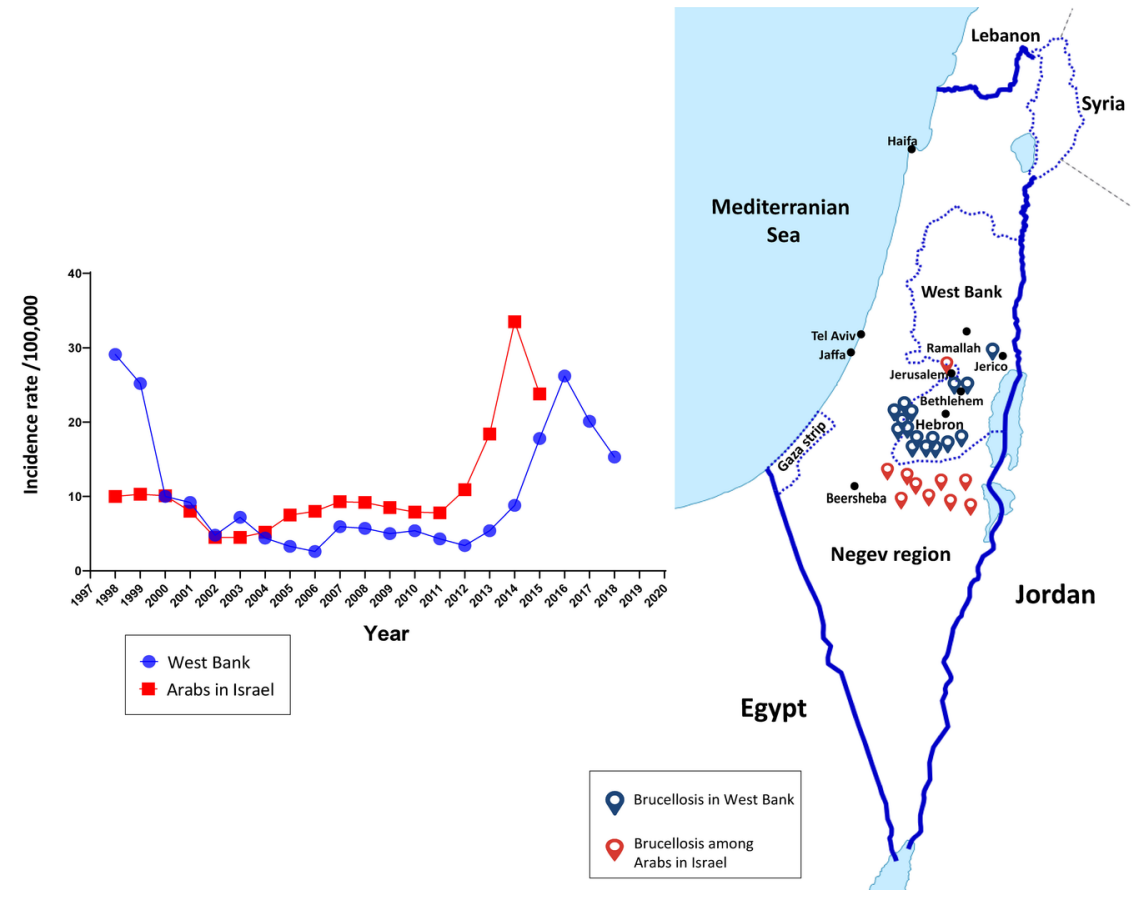

